http://dx.doi.org/ 10.22319/rmcp.v9i1.4518

Nota de investigación

\title{
Desarrollo de un programa en hoja de cálculo para obtener el umbral económico de acaricidas como apoyo en el control de la garrapata Rhipicephalus microplus
}

\section{Development of a spreadsheet program to obtain the economic threshold of acaricides as a support in the control of the cattle tick Rhipicephalus microplus}

Anaid Ireri Hernández García ${ }^{a}$,

Zeferino García Vázquez ${ }^{\mathrm{b}}$, Carlos Gustavo Vázquez- Peláez ${ }^{\mathrm{a}}$, Graciela Guadalupe Tapia Pérez ${ }^{\mathrm{a}^{*}}$

${ }^{a}$ Departamento de Genética y Bioestadística. Facultad de Medicina Veterinaria y Zootecnia. Universidad Nacional Autónoma de México. México.

${ }^{\mathrm{b}}$ Finado.

*Autor de correspondencia: tapiadoctora@gmail.com

\footnotetext{
- Resumen:

El objetivo fue desarrollar un programa de simulación en hojas de cálculo utilizando Excel@, que estima los umbrales económicos de acaricidas usados en el control de garrapata Rhipicephalus microplus. El umbral económico (UE) es la densidad de población del parásito, para tomar la decisión de iniciar una acción de control que impida una pérdida mayor al costo de la intervención. Los parámetros que utiliza el programa son: cantidad de cabezas en la explotación, precio en el mercado por kilo en canal de bovino, y peso promedio
} 
de los bovinos, número de personas que aplican el tratamiento y su salario, número de aplicaciones al año y los insumos de cada producto. Se ejecutaron 10,000 simulaciones con cinco familias de acaricidas diferentes para obtener umbrales económicos utilizando pérdidas de 1.37 y $1.18 \mathrm{~kg}$ por garrapata para dos tipos genéticos de bovinos e $\mathrm{i}=1,2,3 \ldots, 500$ garrapatas, resistentes $(30 \%$ ) y sin resistencia. Los umbrales obtenidos, con $100 \%$ y $70 \%$ de efectividad de los acaricidas fueron 46 y 158 garrapatas respectivamente, la diferencia promedio de pérdida de peso vivo entre $B$. taurus y su cruza con cebú fue de $1.33 \mathrm{~g}$ por garrapata ingurgitada. Al aplicarse un acaricida a una población de garrapatas antes de alcanzar el umbral económico se tienen pérdidas monetarias, por lo que es deseable conocer la resistencia de $R$. microplus y el umbral económico en cada explotación.

- Palabras clave: Umbral económico, Garrapata del ganado, Programa de simulación, Rhipicephalus microplus.

\section{- Abstract:}

The aim of the study was to develop a simulation program in spreadsheets using Excel® to estimate the economic thresholds of acaricides used in the Rhipicephalus microplus tick control. The economic threshold is the population density of the parasite with which the decision is made to initiate a control action that prevents a loss greater than the cost of the intervention. Parameters used by the program are: number, price and weight of cattle, number of people applying the treatment and their income, number of applications per year and the supplies of each product. A total of 10,000 simulations were carried out with five different families of acaricides to obtain economic thresholds using losses of 1.37 and 1.18 $\mathrm{kg}$ per tick for two genetic types of cattle and $\mathrm{i}=1,2,3 \ldots, 500$ ticks, resistance $(30 \%)$ and without resistance. The thresholds obtained by the program, with $100 \%$ and $70 \%$ effectiveness of the acaricides were 46 and 158 ticks respectively, the average difference of live weight loss between $B$. taurus and its cross with zebu was $1.33 \mathrm{~g}$. When the effectiveness of the product decreases, and acaricide is applied to a population of ticks before reaching the economic threshold, there are monetary losses, so it is desirable to know the resistance of $R$. microplus and the economic threshold in each farm.

- Key words: Economic thresholds, Cattle tick, Simulation program, Rhipicephalus microplus.

Recibido el 06/06/2017.

Aceptado el 25/07/2017. 
Se entiende como daño económico a la cantidad de perjuicio que justifica el costo del control artificial, y al umbral económico (UE) como la densidad de población del parásito con la que se toma la decisión de iniciar una acción de control que impida una pérdida mayor al costo de la intervención ${ }^{(1)}$. Aunque se mide como número de parásitos, el UE es realmente el momento que se debe tomar una acción, es decir, los números simplemente son un índice de ese momento; también se le puede llamar umbral de acción ${ }^{(2)}$.

La presencia de la garrapata Rhipicephalus (Boophilus) microplus en los hatos de bovinos causa pérdidas de 0.6 a1.5 g por garrapata ingurgitada en el crecimiento de bovinos en engorda $^{(3)}$ dependiendo de raza. Para México, en 2017 se calcularon las pérdidas potenciales (US\$) en ganado B. taurusde 295'459,145, en B. indicus 179'905,011 y en la cruza 29’365.226 considerando una media de garrapatas ingurgitadas por día de 94.0, 39.9 у 3.3 respectivamente ${ }^{(4)}$.

La estrategia más utilizada para controlar las garrapatas consiste en romper su ciclo biológico a través de la aplicación de tratamientos con acaricidas como organofosforados, inhibidores del desarrollo, piretroides, amidinas e ivermectina a intervalos específicos, pero el uso indiscriminado de estas sustancias ha aumentado la resistencia de este ectoparásito, así como la presencia de residuos químicos en carne y leche, por lo que se han impulsado alternativas que presenten menos daños directos y colaterales a la producción y medio ambiente, como vacunas, otros tratamientos de control biológico y manejo del ganado; estas prácticas son conocidas como Manejo Integrado de Plagas (MIP) ${ }^{(5,6)}$. Desde 1978 en Australia se mostró variación entre razas de resistencia a $R$. microplus: Brahaman $99 \%$, las cruzas de B. indicus y B. taurus de 95 a $97 \%$, Jersey $98 \%$ y la Friesain $85 \%{ }^{(7)}$; esta información ha sido actualizada por otros autores ${ }^{(8-11)}$, pero no se encontró información reciente sobre la disminución de kilogramos de peso vivo causada por la garrapata $R$. microplus en el $B$. indicus puro.

Con objeto de medir, evaluar y tomar decisiones sobre el impacto del deterioro al hospedero y subsecuentes pérdidas económicas, el uso de los umbrales económicos para decidir cuando es económicamente viable aplicar un control puede reducir los costos de producción y tener un impacto ecológico positivo.

La aplicación de las UE en las garrapatas $R$. microplus no es sencilla, debido a que la cantidad de éstas en el cuerpo del bovino es difícil de observar, además son susceptibles a factores como el precio de la carne, el costo y efectividad de la aplicación del acaricida empleado. A pesar de esto, los productores requieren apoyo en la decisión de la elección e implementación del tratamiento más apropiado por su eficacia y precio ${ }^{(12)}$. En años recientes, se han utilizado modelos matemáticos como auxiliares en los análisis de programas más efectivos y económicos de manejo de control de parásitos ${ }^{(12,13,14)}$, por lo que el objetivo del presente estudio fue desarrollar un programa que provea los umbrales económicos de los acaricidas usados contra la garrapata $R$. microplus. 
Desarrollo del programa. El programa se desarrolló con funciones macro en hojas de cálculo de Excel (Figura 1), que consiste principalmente en la obtención de los costos del tratamiento y algunos parámetros con los que se obtienen las pérdidas debidas a no tratar (daño económico).

Figura 1. Diagrama donde se muestra la manera de obtener el costo de tratamiento por hato al año y el daño económico

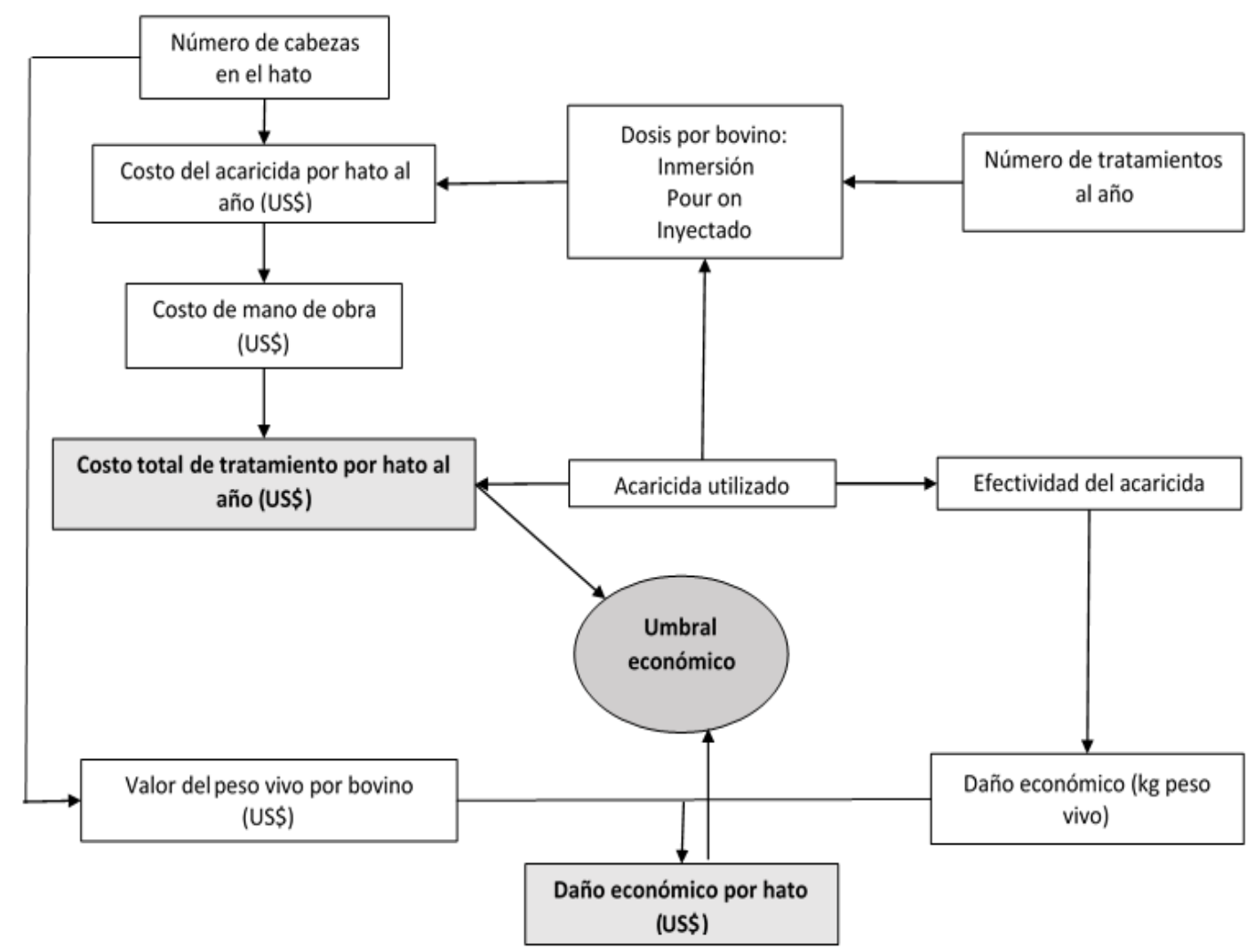

Costo del tratamiento por hato al año. El Cuadro 1 muestra las ecuaciones y variables que lo componen para estimar los costos. El programa utiliza las dosis que indica el laboratorio; el número de cabezas, el peso promedio por bovino; el precio del acaricida y su presentación; número de aplicaciones; número de trabajadores que aplican el producto y el salario promedio por la aplicación del producto; en el caso los productos aplicados por inyección, el costo de las jeringas; y para los aplicados por baño de inmersión, las dimensiones del tanque, la cantidad y precio de $\mathrm{CaO}$ (cal agrícola), incluyéndose una recarga con los valores iniciales por cada $500 \mathrm{~L}$ de desgaste en el tanque. 
Cuadro 1. Ecuaciones para obtener el costo total de tratamiento por hato al año

\begin{tabular}{l} 
Variable \\
\hline Inyectable y Pour on: $^{\text {a }}$ \\
Costo total de tratamiento por bovino al \\
año \\
Costo del material por aplicación al año \\
Costo de mano de obra por tratamiento por \\
bovino al año
\end{tabular}

Costo de la dosis por bovino al año

Costo de la dosis por bovino + costo del material para la aplicación + costo de mano de obra del tratamiento.

Precio de jeringas de $5 \mathrm{ml}$.

(Número de trabajadores * salario del trabajador) / (número de cabezas de ganado*número de tratamientos al año por bovino).

Costo de $1 \mathrm{ml}$ del acaricida $*$ dosis del bovino en mililitros.

Dosis por bovino

(dosis en $\mathrm{ml}$ indicados por el laboratorio* peso promedio del bovino) /unidad de peso indicada por el laboratorio.

Dosis por bovino al año Dosis por bovino*número de tratamientos al año por bovino.

Inmersión

(Primera dilución y recarga: $)^{\mathrm{b}}$

Medidas para el tanque de inmersión

((Largo superior + largo inferior)

$12)^{*}(($ ancho superior + ancho inferior)/2)*altura)*1,000.

Cal agrícola total por tanque

(Cantidad de cal indicada por la farmacéutica* capacidad del tanque) /1,000.

Cantidad de cal por litro de dilución

Cal agrícola total por tanque/ capacidad de tanque.

Costo de la cal agrícola $(\mathrm{CaO})$ total por tanque

(Cal agrícola total por tanque* precio de la cal) /cantidad de cal por costal.

Cantidad de acaricida total por tanque

(Cantidad de acaricida indicada por la farmacéutica/1,000)*(capacidad del tanque/cantidad de agua para diluir el producto indicada por la farmacéutica).

Concentración del acaricida por litro de tanque

Cantidad de acaricida total por tanque/ capacidad del tanque.

Dilución del acaricida por bovino

Concentración del acaricida por litro de tanque*gasto de líquido por bovino en el baño de inmersión. 
Costo del acaricida total por tanque

Costo del acaricida en mililitros

Dosis por bovino

Costo de la dosis por bovino

Costo de la cal agrícola $(\mathrm{CaO})$ por dosis por bovino

Costo total

Daño económico:

Daño económico por pérdidas en peso vivo $(\mathrm{kg})$

Daño económico (US\$)
(Cantidad de acaricida total por tanque*precio del acaricida) / (presentación del producto/1,000)

(Costo del acaricida total para primera dilución + costo del acaricida total para recarga) / (cantidad de la cal agrícola total por tanque para la primera dilución + cantidad de la cal agrícola total por tanque



(Dilución del acaricida por bovino primera dilución + dilución del acaricida por bovino $\left.\operatorname{recarga}^{b}\right)^{*} 1,000$.

Costo del acaricida en mililitros* dosis por bovino.

Cantidad de cal por litro de dilución*costo de la cal agrícola total por tanque.

Costo de la dosis por bovino + mano de obra + material de aplicación.

Número de garrapatas * coeficiente de daño.

(Peso promedio del bovino* precio de venta de peso vivo*(daño económico por pérdidas en peso vivo)) - (peso promedio del bovino*precio de venta de peso vivo*(coeficiente de daño)).

a Derrame sobre el lomo.



Daño económico. El daño económico es la diferencia entre el beneficio y la pérdida debida al tratamiento. Se utiliza la ecuación desarrollada por Southwood y Norton ${ }^{(15)}$. El programa solicita la efectividad del producto, es decir, el porcentaje de población de $R$ microplus eliminada, aquí el usuario puede determinar el grado de resistencia al acaricida.

Umbral económico. La ecuación que se utilizó para obtener los UE fue la propuesta por Norton, Sutherst y Maywald ${ }^{(16)}$ :

$$
\theta=\frac{C}{P d k}
$$

Donde:

$\boldsymbol{\theta}$ es el umbral económico, 
C es el costo del tratamiento por bovino (US\$),

$\mathbf{P}$ es el precio de venta del peso vivo por bovino (US\$),

d es el coeficiente de daño $(\mathrm{kg})$ y

$\mathbf{k}$ es la efectividad del producto empleado para el control de la garrapata.

Se proporcionaron a 10 médicos veterinarios zootecnistas (MVZ) que laboraban en la industria farmacéutica en el área de parasiticidas, tres diferentes valores para cada una de estas variables: producto acaricida, número de aplicaciones al año, peso del bovino, número de cabezas de ganado; los costos (en US\$) en el momento de calcular el umbral de los factores más importantes, precios por kilo de peso vivo, salario actualizado del personal que aplicó el producto; cada uno de estos MVZ obtuvo los costos de tratamiento por su método habitual, y tres umbrales económicos con la ecuación de Norton, Sutherst y Maywald ${ }^{(16)}$. Por otro lado, se introdujeron los mismos valores al programa y los resultados se analizaron mediante una prueba no paramétrica de concordancia de Kendall.

Simulaciones. El Cuadro 2 muestra los parámetros utilizados en el programa. El volumen del tanque de inmersión ${ }^{(17)}$, precio de peso vivo por kilo ${ }^{(18)}$, y coeficiente de daño en dos razas de bovinos (Bos taurus y Bos taurus X Bos indicus) ${ }^{(19)}$ se obtuvieron de fuentes de información publicada; para obtener los demás parámetros se realizó una encuesta de tipo no probabilístico discrecional, entre productores de ganado bovino $(\mathrm{n}=15)$ y MVZ que laboran en la industria farmacéutica veterinaria y que comercializan productos acaricidas para el control de la garrapata $R$. microplus en México $(\mathrm{n}=16)$. Se tomaron los valores más frecuentes (la moda estadística) de los resultados de la encuesta: cabezas de ganado en la explotación (50), peso del bovino (400 kg), número de personas que aplican el tratamiento (2) y su salario (7.94 US\$); en su caso, gasto de líquido por bovino en litros en el baño de inmersión (6 L), con 50 bovinos, el gasto total de dilución del tratamiento en tanque fue de $300 \mathrm{~L}$, costo de jeringas para aplicar ivermectina (0.03 US\$), así como el número de aplicaciones al año de cada sustancia acaricida.

Cuadro 2: Valores empleados en las simulaciones y su fuente de información

\begin{tabular}{lll}
\hline Datos & Valor & Fuente \\
\hline Volumen del tanque de inmersión, $\mathrm{m}^{3}$ & 10,000 & SAGRARPA $^{(17)}$ \\
$\begin{array}{l}\text { Precio de peso vivo por kilogramo de } \\
\text { bovino (US\$) }\end{array}$ & 2.48 & InfoAserca $^{(18)}$ \\
$\quad$ B. taurus & 1.37 & Jonsson $^{(19)}$
\end{tabular}


Coeficiente de $\quad$ B. taurus $x$ B. indicus 1.18

daño

Número de cabezas de ganado

50

Peso del bovino, $\mathrm{kg}$

400

Número de personas que aplican el tratamiento

2

0.1588

Salario de las personas por aplicación del producto (US\$) por bovino

Número de aplicaciones al año

Jeringas $5 \mathrm{ml}$ (US\$/unidad) 5

0.03

Gasto de líquido por bovino en el baño de inmersión (L)

Costo de $\mathrm{CaO}$ (cal agrícola) (US\$/25 kg)
6

5
Productores

MVZ que labora en la industria farmacéutica

Los valores mencionados para validación del programa (Cuadro 2), se pueden modificar en la hoja de cálculo de acuerdo con las condiciones particulares del usuario, exceptuando el coeficiente de daño, dado que sólo es posible obtenerlo mediante experimentación.

Se realizaron 10,000 simulaciones para obtener los umbrales económicos de cinco acaricidas comparándolas en dos grupos de bovinos (B. taurus y B. taurus $x$ B. indicus), con resistencia (30 \%) y sin resistencia de la garrapata a los acaricidas con $\mathrm{i}=1,2,3,4,5, \ldots \ldots, 500$ garrapatas ingurgitadas.

En la prueba no paramétrica de concordancia de Kendal con los cálculos hechos por los MVZ y el programa de simulación se obtuvo un coeficiente de 0.93 , lo que indica un buen consenso entre los resultados.

Los resultados de los umbrales económicos obtenidos a partir de las simulaciones se exponen en el Cuadro 3 y la Figura 2.

Cuadro 3. Umbrales económicos obtenidos a partir de las simulaciones con el programa desarrollado en hojas de cálculo de Excel@

\begin{tabular}{lcccccc}
\hline $\begin{array}{l}\text { Sustancia } \\
\text { activa* }^{*}\end{array}$ & Genotipo & $\begin{array}{c}\text { Resistencia } \\
(\%)\end{array}$ & $\begin{array}{c}\text { Pérdidas } \\
\text { por } \\
\text { garrapata } \\
(\mathbf{g})\end{array}$ & $\begin{array}{c}\text { Daño } \\
\text { económico } \\
\text { (US\$) }\end{array}$ & $\begin{array}{c}\text { Costo de } \\
\text { tratamiento } \\
\text { total anual } \\
\text { por hato } \\
\text { (US\$) }\end{array}$ & $\begin{array}{c}\text { Umbral } \\
\text { económico } \\
\text { (número de } \\
\text { garrapatas) }\end{array}$ \\
\hline Amitraz & B. taurus & 0 & 29.21 & 10.35 & 122.94 & 23 \\
& & 30 & 29.34 & 10.72 & & 77
\end{tabular}




\begin{tabular}{|c|c|c|c|c|c|c|}
\hline & B. taurus & 0 & 29.50 & 15.58 & & 25 \\
\hline & $\begin{array}{c}x B . \\
\text { indicus }\end{array}$ & 30 & 31.15 & 21.67 & & 88 \\
\hline \multirow[t]{4}{*}{ Cipermetrina } & B. taurus & 0 & 46.99 & 77.73 & 246.52 & 37 \\
\hline & & 30 & 49.15 & 88.05 & & 129 \\
\hline & B. taurus & 0 & 48.38 & 88.71 & & 41 \\
\hline & $\begin{array}{c}x B . \\
\text { indicus }\end{array}$ & 30 & 49.91 & 96.21 & & 141 \\
\hline \multirow[t]{4}{*}{ Coumafos } & B. taurus & 0 & 41.91 & 55.27 & 203.21 & 33 \\
\hline & & 30 & 43.05 & 60.10 & & 113 \\
\hline & B. taurus & 0 & 42.48 & 62.05 & & 36 \\
\hline & $\begin{array}{c}x B . \\
\text { indicus }\end{array}$ & 30 & 43.90 & 68.13 & & 124 \\
\hline \multirow[t]{4}{*}{ Fluazurón } & B. taurus & 0 & 115.57 & 632.20 & 997.58 & 91 \\
\hline & & 30 & 117.73 & 657.25 & & 309 \\
\hline & B. taurus & 0 & 118.00 & 664.82 & & 100 \\
\hline & $\begin{array}{c}x B . \\
\text { indicus }\end{array}$ & 30 & 119.3 & 680.13 & & 337 \\
\hline \multirow[t]{4}{*}{ Ivermectina } & B. taurus & 0 & 44.45 & 60.65 & 234.95 & 35 \\
\hline & & 30 & 47.63 & 80.72 & & 125 \\
\hline & B. taurus & 0 & 47.20 & 83.10 & & 40 \\
\hline & & 30 & 48.50 & 89.28 & & 137 \\
\hline
\end{tabular}

*Las dosis por bovino se calcularon siguiendo las especificaciones técnicas que el laboratorio indica en cada producto.

** El costo de tratamiento total por hato al año incluye: costo de material y costo de mano de obra.

Figura 2. Umbrales económicos el B. taurus y $B$. taurus x B. indicus en dólares americanos (US\$) con el número de garrapatas ingurgitadas con $30 \%$ de resistencia ( $70 \%$ eficacia) y sin resistencia (100\% eficacia) por hato al año en cada una de las sustancias activas 


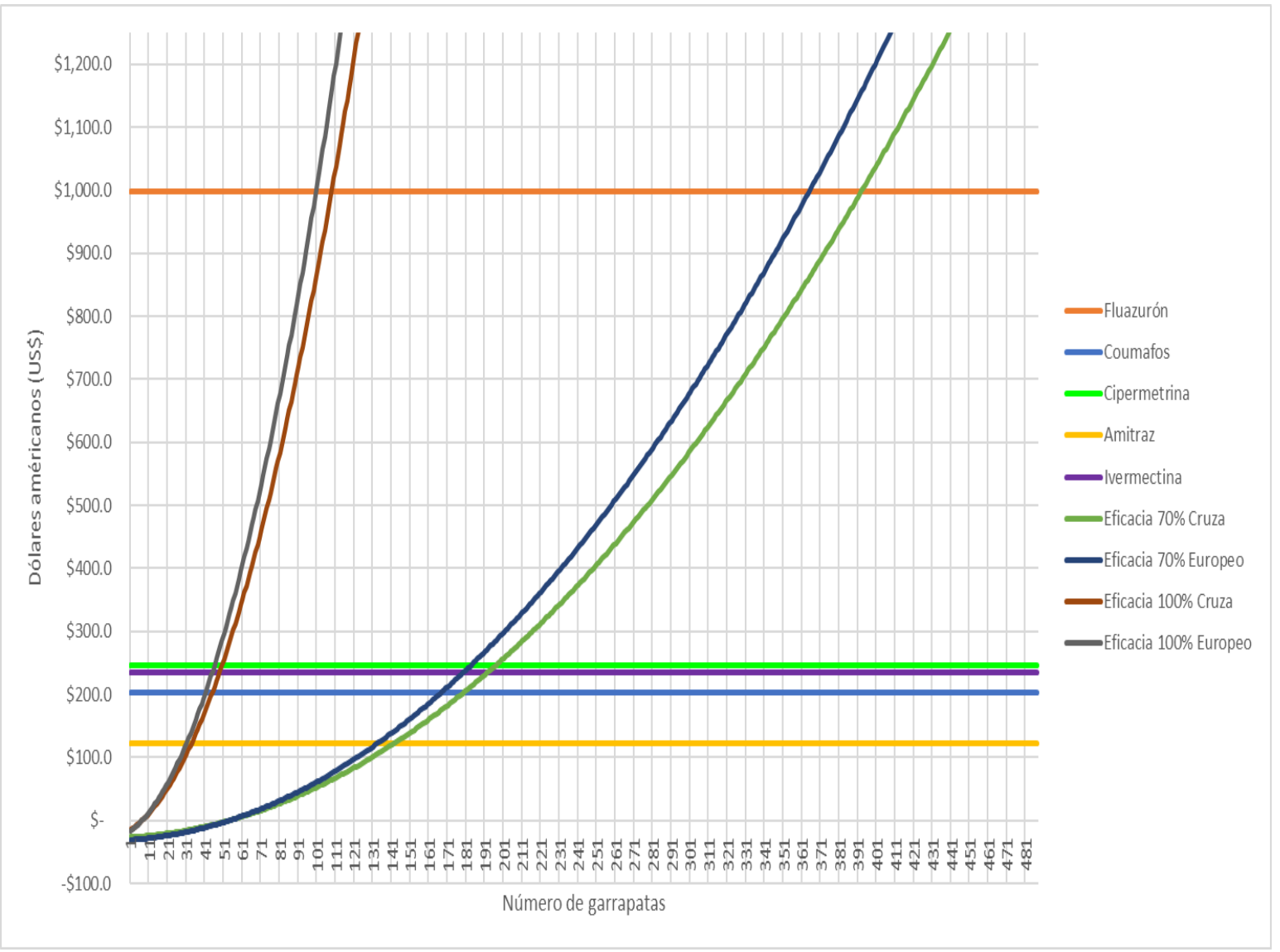

El costo es el factor más importante para la elección de un producto acaricida, y se debe, en gran parte, al equipo necesario para las aplicaciones, que se han estimado en 78 y $88 \%{ }^{(20)}$; y a la dosis necesaria para eliminar la mayor parte de las garrapatas. De las cinco sustancias activas de acaricidas analizados los costos fueron: el fluazurón US\$ 997.58, la cipermetrina, 246.52 US\$, ivermectina 234.95 US\$, coumafos 203.21, el amitraz tuvo el menor costo de tratamiento US\$122.94.

La cantidad de cal agrícola $(\mathrm{CaO})$ requerida para el uso del producto, aumentó el costo de tratamiento en coumafos comparado con amitraz; ambos productos se aplican mediante baño de inmersión y la dosis por bovino calculada es menor en el primero $(5 \mathrm{ml})$ que en el segundo $(9.6 \mathrm{ml})$, sin embargo, el costo de tratamiento total fue mayor en coumafos que en amitraz (US\$ $203.21 v s$ US\$ 122.94 respectivamente), ya que el primero utiliza mayor cantidad de $\mathrm{CaO}$.

En la práctica, es el costo unitario, no el costo del tratamiento lo que define la elección de un producto acaricida, pues el usuario no suele considerar el volumen y la dosificación, de modo que adquiere envases que aparentan ser más baratos, pero que en términos de dosis, son en realidad más costosos, por lo que en este rubro la intervención del especialista es crucial. 
Otro elemento importante que considerar en el costo de tratamiento, es si se cuenta o no con baño de inmersión en las instalaciones de la explotación; si es así, el producto por elegir sería el amitraz, después el coumafos y la cipermetrina que tienen costos parecidos entre ellos, pero más altos que el amitraz. En caso de no tener el baño de inmersión, la ivermectina sería la elección, -aunque no es precisamente un acaricida, se incluyó en el estudio pues se utiliza como auxiliar en el control de la garrapata como lo indican los laboratorios que lo producen, pero implica mayor manejo, pues se requiere estimar el peso de cada animal-. El fluazurón (familia fenilurea) de aplicación pour on o aplicación sobre el lomo, elegido en este estudio resultó costoso, pero pueden existir en el mercado otros productos de menor precio que pueden añadirse en la hoja de cálculo. La ventaja del programa es que se puede cambiar en la carátula el producto y la vía de aplicación para calcular su costo, si se desea aplicar por esta vía.

Diferentes autores han expuesto las pérdidas generadas por no aplicar tratamientos contra las garrapatas; en Paraguay encontraron que las razas Friesian y Charolais presentaban en promedio 1,760 garrapatas mayores a $4 \mathrm{~mm}$ de largo, disminuyendo la salud y la ganancia de peso $^{(21)}$. En Gambia, encontraron pérdidas de hasta $8 \mathrm{~kg}$ menos de peso que en animales tratados (7.9 vs $15.9 \mathrm{~kg}$ respectivamente), en un periodo de 11 meses en ganado nativo ${ }^{(22)}$.

Los resultados de las simulaciones del daño por pérdidas en peso vivo causadas por la garrapata, en $B$. taurus $X$ B. indicus y $B$. taurus puro, así como para animales con y sin resistencia, sólo son cálculos que el programa obtiene de acuerdo con los coeficientes de daño, publicados por Jonsson ${ }^{(19)}$. La resistencia (como efectividad del producto acaricida), también se puede cambiar en la carátula de las hojas de cálculo, no así los coeficientes de daño. Otros estudios con Amblyoma hebraeum y R. appendiculatus ${ }^{(23,24)}$ también informaron que las razas cebú (B. indicus) presentaron mayor resistencia a garrapatas en relación con las razas europeas (B. taurus). Hacen falta estudios sobre coeficientes de daños en el ganado bovino mexicano.

El umbral económico es el momento oportuno de aplicar el control químico con el fin de impedir el daño producido por las garrapatas, y también evitar un gasto monetario innecesario por el tratamiento. En la Figura 2, el UE es la intersección entre las líneas del daño económico y las del costo del tratamiento anual por bovino. En Australia, en 1983 se estimó un umbral económico de 158 garrapatas ingurgitadas por animal por día, aunque no se menciona el acaricida que se empleó para este cálculo ${ }^{(16)}$, y en el 2001 utilizando el programa de Sutherst $^{(25)}$ se estimó un umbral económico de 100 garrapatas por cabeza $^{(26)}$; en Paraguay con una mezcla organofosforado/piretroides se calculó UE de 53 garrapatas por animal en 1988/1989 y 54 en 1990/1991 con las razas Santa Gertrudis, Brahaman y Nelore ${ }^{(27)}$. En un manual técnico para productores se recomienda comenzar a tratar cuando se observen más de 30 garrapatas, sin embargo no es claro cuál es el argumento con el que se obtuvo este número $^{(28)}$. 
En este trabajo se encontró un aumento del umbral económico promedio de 112 garrapatas con una resistencia de $30 \%$ a los acaricidas. Cuando los niveles efectividad de los acaricidas disminuyen, los beneficios debidos al tratamiento se alcanzan con un mayor número de garrapatas, al aplicarse el acaricida a una población de garrapatas resistente antes de alcanzar el umbral económico se originan pérdidas monetarias. Por ejemplo, en una explotación de 70 cabezas, esta diferencia (112 garrapatas) causaría una pérdida de $155 \mathrm{~g}$ por bovino en promedio, perdiéndose en total 10,850 g que representan 26.91 US\$ en cada aplicación. La resistencia puede ser diferente dentro de la misma región geográfica ${ }^{(29-32)}$, por lo cual se recomienda enviar periódicamente muestras de garrapatas de la explotación a laboratorios donde se realicen diagnósticos de resistencia.

Hoy en día se sabe que la estrategia más deseable para mantener la calidad ambiental y mejorar las ganancias, es el manejo de la garrapata y no su erradicación. En general, un número bajo de garrapatas no causan un daño importante; de hecho, mantener bovinos en la explotación sin tratar con acaricidas, podría ser deseable para mantener poblaciones de garrapatas susceptibles (refugio), que al hibridarse con las resistentes, puedan eliminarse fácilmente ${ }^{(33)}$; pero para ello es indispensable analizar en cada explotación, la cantidad de garrapatas presentes por bovino, ponderando el impacto económico de éstas sobre los costos corrientes de gestión. Los umbrales también proporcionan un punto de partida para conocer el estatus de la explotación, son números únicos, específicos para cada una. En este trabajo se consideraron dos genotipos de bovinos, la cruza de estos es más tolerante al daño, y podría ser un punto de partida para dejar estos animales como 'refugio' proveedor de garrapatas susceptibles ${ }^{(34)}$.

Los umbrales son valiosos, pero saber usarlos es tan importante como calcularlos; es deseable que el productor sea asesorado por un especialista para analizar las implicaciones de los resultados. Mientras más se comprende la situación y el crecimiento de las poblaciones de garrapatas en cada explotación, se tomarán mejores decisiones que aporten más ganancias para el productor.

Los programas de simulación en investigación son actualmente la base para el establecimiento de las estrategias del control de la garrapata $R$. microplus, que permiten tomar decisiones que reduzcan el uso de acaricidas y pérdidas en la producción; sin embargo, estos deben de ser probados de manera experimental antes de que se usen para el tratamiento contra la garrapata en el ganado infestado. 


\section{- Literatura citada}

1. Stern VM, Smith RF, Van den Bosch R, Hagen KS. The integrated control concepts. Hilgardia 1959;(29):81-101.

2. Pedigo LP. Entomology and pest management. Second ed. New Jersey, USA: Prentice Hall Pub; 1996.

3. Wambura PN, Gwakisa PS, Silayo RS, Rugaimukamu EA. Breed-associated resistance to tick infestation in Bos indicus and their crosses with Bos taurus. Vet Parasitol 1998;(77):63-70.

4. Rodriguez VR, Grisi L, Pérez LA, Silva VH, Torres AJ, Fragoso SH, Romero SD, et al. Evaluación del impacto potencial de los parásitos del ganado bovino en México. Revisión. Rev Mex Cienc Pecu 2017;8(1):61-74. http://dx.doi.org/10.22319/rmcp.v8i1.4305

5. Rodríguez VR, Rosado AA, Ojeda CMM, Pérez CCL, Trinidad MI, Bolio GME. Control integrado de garrapatas en la ganadería bovina. Eco Rec Agro 2014;1(3):295-308.

6. Rodríguez VR, Rosado AA, Basto EG, García VZ, Rosario CR, Fragoso SH. Manual técnico para el control de garrapatas en el ganado bovino. Instituto Nacional de Investigaciones Forestales Agrícolas y Pecuarias. Centro Nacional de Investigaciones Disciplinarias en Parasitología Veterinaria. Publicación Técnica. 2006.

7. Utech KBW, Wharton RH, Kerr JD. Resistance to Boophilus microplus (Canestrini) in different breeds of cattle. Aust J Agric Res 1978;(29):885-895.

8. Jonsson NN. The productivity effects of cattle tick (Boophilus microplus) infestation on cattle, with particular reference to Bos indicus cattle and their crosses. Vet Parasitol 2006;(137):1-10.

9. Da - Silva AM, Mello AM, Correia ARL, De- Sena OMC, Barioni JW. Artificial infestation of Boophilus microplus in beef cattle heifers of four genetic groups. Genet Mol Biol 2007;30(4):1150-1155.

10. Alonso DMA, López SBJ, Leme MLAC, Rodríguez VRI. Infestación natural de hembras de Boophilus microplus Canestrini, 1887 (Acari: Ixodidae) en dos genotipos de bovinos en el trópico húmedo de Veracruz, México. Rev Vet Mex 2007;38(4):503-509.

11. Shyma KP, Prakash GJ. Breeding strategies for tick resistance in tropical cattle: a sustainable approach for tick control. J Parasit Dis 2015;39(1):1-6.

12. Liang J, Tang S. Optimal dosage and economic threshold of multiple pesticide applications for pest control. Math Comput Model 2010;51(5-6):487-503. 
13. Tang S, Cheke RA. Models for integrated pest control and their biological implications. Math Biosci 2008;215(1):115-215.

14. Tang S, Xiao Y, Cheke RA. Dynamical analysis of plant disease models with cultural control strategies and economic thresholds. Math Comput Simulat 2010;(80):894-921.

15. Southwood TRE, Norton GA. Economic aspects of pest management strategies and decisions. Insects: Studies in population management. In: Geier PE et al, editors. Canberra, Australia: Ecol Soc Aust 1973:168-84.

16. Norton GA, Sutherst RW, Maywald GF. A framework for integrating control methods against the cattle tick, Boophilus microplus in Australia. J Appl Ecol 1983;20(2):489505.

17. SAGARPA (Secretaría de Agricultura, Ganadería, Desarrollo Rural, Pesca y Alimentación). Art 24 III b) iv. Baño de inmersión. En Acuerdo por el que se establece la campaña nacional para el control de la garrapata Boophilus spp. Diario Oficial (Primera Sección). México. 2012.

18. INFOASERCA. Arribos de bovinos en pie. 2017. http://www.infoaserca.gob.mx/ bovinos/gb_rastro.asp. Consultado $11 \mathrm{Jul}, 2017$.

19. Jonsson NN. The productivity effects of cattle tick (Boophilus microplus) infestation on cattle, with particular reference to Bos indicus cattle and their crosses. Vet Parasitol 2006;(137):1-10.

20. McLeod RS. Costs of major parasites to the Australian livestock industries. Int J Parasitol 1995;25(11):1363-1367.

21. Quinlan JF, Scarone CA, Laneri JL. Cattle tick identification and seasonal variation in infestation rates in Paraguay. Trop Anim Hlth Prod 1980;12:259-264.

22. Mattioli RC, Jaitner J, Bah M. Efficiency and cost of strategic use of acaricide for tick control in N'Dama cattle in The Gambia. Med Vet Entomol 1999;(13):33-40.

23. Norval RAI, Sutherst OG, Jorgensen, Gibson JD, Kerr JD. The effect of the Bont tick (Amblyomma hebraeum) on the weight gain of Africander steers. Vet Parasitol 1989;(33):329-341.

24. De Castro JJ, James AD, Minjauw B, Di Giulio GU, Permin A, Pegram RG, Chizyuka HGB, Sinyangwe P. Long-term studies on the economic impact of ticks on Sanga cattle in Zambia. Exp Appl Acarol 1997;(21):3-19. 
25. Sutherst RW, Maywald GF, Kerr JD, Stegemen DA. The effect of the cattle tick Boophilus microplus on the growth of Bos indicus $x$ Bos taurus steers. Aust J Agric Res 1983;(34):317-327.

26. Jonsson NN, Davis R, De Witt M, An estimate of the economic effects of cattle tick (Boophilus microplus) infestation on Queensland dairy farms. Aust Vet J 2001;(79):826831.

27. Brizuela CM, Ortellado CA, Sánchez TI, Osorio O, Walker AR. Formulation of integrated control of Boophilus microplus in Paraguay: analysis of natural infestations. Vet Parasitol 1996;(63):95-108.

28. Rodriguez VRI, Torres AJF, Ramirez CG, Rosado AJA, Aguilar CAJ, Ojeda CMM, Bolio GME. Control de parásitos internos y externos que afectan al ganado bovino en Yucatán, México. CONACYT, UADY. México. 2011.

29. Fernández SA, Rodríguez VRI, Alonso DMA, Basurto CH. Ivermectin resistance status and factors associated in Rhipicephalus microplus (Acari: Ixodidae) populations from Veracruz, Mexico. Vet Parasitol 2012;(190):210-215.

30. Fernández SA, Rodríguez VRI, Alonso DMA. First report of a Rhipicephalus microplus tick population multi-resistant to acaricides and ivermectin in the Mexican tropics. Vet Parasitol 2012;(183):338-342.

31. Fernández SA, Rodríguez VRI, Alonso DMA. Resistance of Rhipicephalus microplus to amitraz and cypermethrin in tropical cattle farms in Veracruz, Mexico. J Parasitol 2012;98(5):1010-1014.

32. Rodríguez-Vivas I, Pérez-Cogollo LC, Rosado-Aguilar JA, Ojeda-Chi MM, TrinidadMartinez I, Miller RJ et al. Rhipicephalus (Boophilus) microplus resistant to acaricides and ivermectin in cattle farms of Mexico. Braz J Vet Parasitol 2014;23(2):13-122. Doi:http://dx.doi.org/10.1590/S1984-29612014044.

33. Tapia-Perez G, Garcia-Vazquez Z, Montaldo H, George J. Inheritance of resistance to flumethrin in the mexican aldama strain of the cattle tick Boophilus microplus (Acari: Ixodidae). Exp Appl Acarol 2003;31(3):135-149.

34. Tapia PGG. Manejo de la resistencia a acaricidas en Ripicephalus (Boophilus) microplus. En: Epidemiología de enfermedades parasitarias en animales domésticos. Quiroz RH, et al, editores. $1^{\text {a }}$ ed. México. 2011. 